\title{
Eltern sind Führungskräfte
}

\section{Familien stehen heute vor zahlreichen Herausforderungen - Gruppenangebote können beim Umgang mit Kindern qualifizieren}

Wolfgang Widder

Wolfgang Widder ist selbstständiger Supervisor, Fortbildner und Berater für Organisationsentwicklung. E-Mail wolfgang.widder@widder-weiler.de
Wenn aus einem Paar eine Familie wird, muss der Alltag neu organisiert werden. Elternbildung ohne erhobenen Zeigefinger kann dabei helfen.

Eltern haben es schwer heutzutage; darüber herrscht meist Einigkeit. Da werden allerhand familienwidrige Umstände genannt: materielle Bedingungen, Arbeitszeitregelungen, fehlende Kinderbetreuungsinstitutionen, Kinderfeindlichkeit u. a.

Eltern haben es auch schwer, weil sie als Schlüssel für unseren gesellschaftlichen Gesamterfolg gesehen werden: Sie sollen viele Kinder, gesunde Kinder, erfolgreiche Kinder, makellose Kinder, schlanke Kinder, sozial kompetente Kinder produzieren. Damit die Demografie stimmt, die Kriminalität sinkt, die Wirtschaft Erfolg hat usw.

Damit sie diesen Ansprüchen einerseits gerecht werden, sie andererseits aber auch zurückweisen können, brauchen Eltern gute Qualifikationen.

\section{Zwölf Herausforderungen für Eltern}

Elternschaft ist einerseits - bei vielen Glückserlebnissen mit Kindern - mit etlichen quasi-natürlichen Herausforderungen verbunden., die schon immer oder zumindest schon lange bestehen. Sieben solche Herausforderungen sind meines Erachtens wesentlich:

1. Wenn junge Paare Eltern werden, verändert sich ihre Beziehungsdynamik zwischen Mann und Frau.

2. Eltern müssen viele Jahre ihres Lebens mit der permanenten Verantwortung für die Existenz und Entwicklung eines bedürftigen Wesens klar kommen.

3. Eltern müssen ertragen lernen, dass sich so manche Erwartung an ihr Kind nicht erfüllt.

4. Eltern haben immer wieder neue, oft überraschende innerfamiliäre Konflikte zu bewältigen.
5. Als Familie sind Wege in die Gesellschaft und ihre Institutionen $\mathrm{zu}$ finden: Kindergarten, Freundeskreise, Schulen, Vereine etc.

6. Eltern müssen es ertragen, dass Kinder sich als Heranwachsende aus der Familie lösen - oder dass sie sich nicht so recht vom Elternhaus lösen wollen!

7. Und sie haben damit fertig zu werden, dass aus Eltern mit erwachsenen Kindern wieder Paare werden.

$\mathrm{Zu}$ diesen sieben alten kommen heute fünf neue Herausforderungen hin$\mathrm{zu}$ :

8. Erhöhte Glücks- und Perfektionsansprüche: Neben dem perfekten Kind soll man als Partner toll und im Beruf erfolgreich sein. Die verbreitete Jederist-seines-Glückes-Schmied-Ideologie schiebt Misserfolge und Schicksalsschläge gerne dem Einzelnen in die Schuhe.

9. Die permanenten BildschirmmedienInvasionen: Sie prägen und gefährden einerseits die Kinder und das Familienleben selbst, andererseits vervielfachen sie durch diverse Sendungen Ansprüche und Schuldvorwürfe gegen Eltern. Über die Suchtgefahren und anderen negativen Auswirkungen, die mit dem grassierenden zeitlich überbordenden Konsum der Bildschirmmedien an sich verbunden sind, sowie die ethischen Dimensionen (Zugang zu Gewaltdarstellungen und Pornografie) ist genug bekannt.

10. Natürlich stellen auch die heute häufigeren Trennungen und Familienneubildungen besondere Herausforderungen für Eltern dar. Dabei sind die Trennungen natürlich auch durch unsere Sinn- und Glückserwartungen an die Partnerschaft bedingt, siehe Punkt 8. Aber wir können und wollen natürlich auch nicht zurück zur Ehestiftung durch die Eltern!

11. Des weiteren sehe ich eine Schwierigkeit für eine Vielzahl von Eltern mit 
Migrationshintergrund und bikulturellen Partnerschaften in der Auseinandersetzung mit Wertedifferenzen innerhalb der Familie oder zwischen Herkunftsland und unserer Kultur.

12. Und als letztes möchte ich die Auseinandersetzung mit den allgegenwärtigen penetranten Konsumangeboten gerade für Kinder nennen, die freilich eng mit unserem fragwürdigen wachstumsorientierten, expansiven Lebensstil und Gesellschaftsmodell verflochten sind.

\section{Wie Eltern lernen, Eltern zu sein}

Menschen, die zu Eltern werden, müssen auf viele Fragen rasch Antworten finden und ihr Leben neu organisieren. Doch wo können sie das lernen? Vier Möglichkeiten gilt es zu unterscheiden:

- Da ist zunächst das Vorbild der eigenen Eltern, die eigene gelungene wie misslungene selbst erlebte Erziehung.

- Dann kommt das »Learning by doing “ - wobei wir wissen, dass auch »eigentlich « Falsches oft erfolgreich ist, wenigstens kurzfristig, deshalb wird es ja gelernt.

- Des weiteren lernen Eltern durch den Austausch mit anderen Eltern, durch die direkte Beobachtung aktuellen Handelns anderer Eltern oder anderer Personen, die mit Kindern umgehen.

- Und zuletzt kommt das Lernen durch Expertenhinweise oder fachliche Hilfe aller Art. Wenn es beispielsweise um die konstruktive Verarbeitung massiver eigener schlechter Erfahrungen geht, ist das ohne fremde Hilfe oft kaum möglich und die Hinzuziehung von Fachleuten das Richtige.

Eltern qualifizieren sich also im Zuge ihrer Lebensführung selbst. Das wird auch in Zukunft so sein, wenngleich das durch unser sich ständig beschleunigendes gesellschaftliches Entwicklungstempo erschwert werden wird.

Für motivierte Eltern gibt es im Bedarfsfall und bei Interesse zahlreiche Hilfe- und Qualifizierungsangebote. Zudem sind sie oft selbst aktiv, um informelle Eltern-Netzwerke zu bilden oder sich ihnen anzuschließen. Auch für Familien mit bestehenden gravierenden sozialen oder gesundheitlichen Problemen oder Behinderungen hält unser Jugendhilfe- und Gesundheitssystem einige Instrumente bereit, auch wenn manches in den letzten Jahren zurückgefahren wurde oder noch verbessert werden muss.

Eine große Gruppe von Eltern ist mangels Dramatik - nicht im Blickfeld der Öffentlichkeit. Ihre Selbsthilfekräfte sind in Krisensituationen überstrapaziert, sie fühlen sich teilweise alleingelassen. Sie $\mathrm{zu}$ unterstützen, sollte gesellschaftliche Aufgabe sein. Das Prinzip Vorbeugung bammen, Ärzten und Ärztinnen, Erzieherinnen, Logopäden, Sozialarbeitern, Lehrerinnen, Therapeuten etc. -, kommt entscheidende Bedeutung zu, wenn man Elterngruppenarbeit flächendeckend zum Erfolg führen will. Sie müssen diese Qualifizierungsangebote kennen und verstehen und als Vermittler agieren. Drei weitere Erfolgsfaktoren für eine qualifizierte Elterngruppenarbeit kommen hinzu:

\section{"Eltern qualifizieren sich zunächst im Zuge ibrer Lebensführung selbst «}

spielt auch für diese, sicher sehr heterogene Gruppe von Eltern eine Rolle.

Am Rande möchte ich bemerken, dass eine weitere Gruppe nicht unbedeutend ist: die Großeltern. Ihre Rolle als Unterstützer von Familie ist nicht zu unterschätzen und auch ihre Qualifikation sollte systematisch gefördert werden.

\section{Elterngruppen als Instrument zur Qualifizierung und zur Förderung von Eltern- Netzwerken}

Neben den bestehenden professionellen Beratungsangeboten für Einzelpersonen oder die einzelne Familie wird eine Möglichkeit für die Elternqualifizierung leichtfertig unterschätzt: die Elterngruppenarbeit. Die systematische Elterngruppenarbeit mit vielen Übungselementen, wie sie da oder dort schon verschiedentlich angeboten wird, ist meines Erachtens das beste Instrument, Eltern über die Jahre immer wieder in den verschiedenen Entwikklungsphasen und Herausforderungen zu unterstützen und gleichzeitig kräftige Impulse zu geben, sich auszutauschen und im Alltag zu unterstützen. Wenn Eltern sich miteinander auseinandersetzen und sich solidarisieren, bestehen beispielsweise bessere Chancen, die Bildschirmmedien-Invasionen einigermaßen zu kontrollieren. Auch die Entlastungsfunktion von sich austauschenden Gruppen ist nicht zu unterschätzen: »Aha, es geht nicht nur uns so! «

Einer systematischen professionellen Vorfeld- und Begleitarbeit mit den Institutionen und Berufsgruppen, die mit Eltern frühzeitig Kontakt haben - also He-
- ein schlüssiges Gesamtkonzept, das sowohl lebensphasen- als auch problembezogene Gruppenangebote umfasst und das kontinuierlich ins Blickfeld aller Beteiligten gerückt wird

- Gruppenkonzepte, die neben gemeinsamer Besprechung schwieriger Familiensituationen klare Trainingsschwerpunkte haben

- eine gut ausgebildete und qualifiziert unterstützte Leitergruppe.

Die Träger von Kindertagesstätten wären, je nach lokaler Ausgangssituation und möglichen Kooperationspartnern auf Gemeindeebene, die richtigen Akteure, solche Angebote zu stimulieren, zu konzipieren und umzusetzen.

\section{Resümee}

Eltern sind die Führungskräfte einer Familie. Wir können uns damit abfinden, dass es halt solche und solche gibt. Wie im Betrieb, im Verein, in der Politik. Wir können aber auch für die Position werben, dass lebenslanges Lernen uns und unsere Kinder bereichert. Dann ist ElternSein leichter - und hat die Chance, zum Sinn-Vollsten und Beglückendsten im Leben zu gehören! 
"Bevor ich heiratete, hatte ich sechs Theorien über Kindererziehung. Jetzt habe ich sechs Kinder und keine Theorie."

John Wilmot, englischer Schriftsteller (1647-1680)

"Kindererziehung ist ein Beruf, wo man Zeit zu verlieren verstehen muss, um Zeit zu gewinnen."

Jean Jacques Rousseau, französischer Schriftsteller (1712-1778)

"Wer in Gegenwart von Kindern spottet oder lügt, begeht ein todeswürdiges Verbrechen.«

Marie von Ebner Eschenbach, österreichische Schriftstellerin (1830-1916)

"Zuerst lieben die Kinder ihre Eltern. Nach einer gewissen Zeit fällen sie ihr Urteil über sie. Und selten, wenn überhaupt je, verzeihen sie ihnen."

Oscar Wilde, irischer Schriftsteller (1854-1900)

"Wenn die Ohrfeige keinem schadet, warum führen wir sie nicht unter Erwachsenen ein? "

Dorle Marx, Rechtsanwältin und SPD-Politikerin (geb. 1957)

"Es gibt kein problematisches Kind, es gibt nur problematische Eltern."

Alexander S. Neill, englischer Pädagoge (1883-1973)

"Wie töricht, den Kindern irgendwelche Freuden zu entziehen, in der Meinung, sie dadurch für die Härte des Lebens zu festigen! Als ob ein schlechtes Mittagbrot leidlicher schmeckte, weil auch das Frühstück verdorben warlı

Alfred Grünewald, österreichischer Schriftsteller (1884-1942) 